\title{
THE METHOD OF LEAST SQUARES FOR BOUNDARY VALUE PROBLEMS
}

\author{
BY \\ JOHN LOCKER
}

\begin{abstract}
The method of least squares is used to construct approximate solutions to the boundary value problem $\tau f=g_{0}, B_{i}(f)=0$ for $i=1, \ldots, k$, on the interval $[a, b]$, where $\tau$ is an $n$th order formal differential operator, $g_{0}(t)$ is a given function in $L^{2}[a, b]$, and $B_{1}, \ldots, B_{k}$ are linearly independent boundary values. Letting $H^{n}[a, b]$ denote the space of all functions $f(t)$ in $C^{n-1}[a, b]$ with $f^{(n-1)}$ absolutely continuous on $[a, b]$ and $f^{(n)}$ in $L^{2}[a, b]$, a sequence of functions $\xi_{t}(t)(i=1,2, \ldots)$ in $H^{n}[a, b]$ is constructed satisfying the boundary conditions and a completeness condition. Assuming the boundary value problem has a solution, the approximate solutions $f_{i}(t)=\sum_{j=1}^{i} a_{j}^{i} \xi_{j}(t)(i=1,2, \ldots)$ are constructed; the coefficients $a_{j}^{i}$ are determined uniquely from the system of equations

$$
\sum_{j=1}^{l}\left(\tau \xi_{j}, \tau \xi_{l}\right) a_{j}^{l}=\left(g_{0}, \tau \xi_{l}\right), \quad l=1, \ldots, i
$$

where $(f, g)$ denotes the inner product in $L^{2}[a, b]$. The approximate solutions are shown to converge to a solution of the boundary value problem, and error estimates are established.
\end{abstract}

1. Introduction. In this paper we utilize the method of least squares to construct approximate solutions to linear nonselfadjoint boundary value problems. The method of least squares has a long history of widespread application, and it has been thoroughly treated by Mihlin [6] and Mihlin and Smolitskiy [8]. Petryshyn [9] has studied it as a direct method for solving linear equations in Hilbert space. Recently Varga [11] and Ciarlet, Schultz, and Varga [1], [2] have used the closely related Rayleigh-Ritz method to yield numerical methods of high-order accuracy for boundary value problems.

For a closed interval $[a, b]$ let $S$ be the real Hilbert space of all real-valued functions $f(t)$ which are square-integrable on $[a, b]$. The usual inner product and norm in $S$ are denoted by $(f, g)$ and $\|f\|$, and we denote convergence in $S$ by $f_{i} \rightarrow f$. For any operator $L$ in $S$ we denote its domain, range, and null space by $\mathscr{D}(L), \mathscr{R}(L)$, and $\mathscr{N}(L)$, respectively.

Received by the editors February 3, 1970.

AMS 1969 subject classifications. Primary 6520, 6562; Secondary 4630, 4760.

Key words and phrases. Method of least squares, boundary value problems, formal differential operator, boundary values, differential operator in Hilbert space, equivalent norms, complete sequences, Gram-Schmidt process, nonhomogeneous boundary conditions.

Copyright (C) 1971, American Mathematical Society 
For an $n$th order formal differential operator

$$
\tau=\sum_{i=0}^{n} a_{i}(t)\left(\frac{d}{d t}\right)^{i}
$$

we assume that the coefficients $a_{i}(t)$ are real-valued functions belonging to $C^{\infty}[a, b]$, and that $a_{n}(t) \neq 0$ on $[a, b]$. Given an $n$th order formal differential operator $\tau$ and a set of $k$ linearly independent boundary values

$$
B_{i}(f)=\sum_{j=0}^{n-1} \alpha_{i j} f^{(j)}(a)+\sum_{j=0}^{n-1} \beta_{i j} f^{(j)}(b), \quad i=1, \ldots, k,
$$

we define a differential operator $L$ in $S$ as follows: let $H^{n}[a, b]$ be the linear subspace of $S$ consisting of all functions $f(t)$ in $C^{n-1}[a, b]$ with $f^{(n-1)}$ absolutely continuous on $[a, b]$ and $f^{(n)}$ in $S$, and let

$$
\mathscr{D}(L)=\left\{f \in H^{n}[a, b] \mid B_{i}(f)=0, i=1, \ldots, k\right\}, \quad L f=\tau f .
$$

For a fixed function $g_{0}(t)$ in $S$ we consider the linear boundary value problem

$$
L f=g_{0} .
$$

To solve equation (1) by the method of least squares, we choose a sequence of functions $\xi_{i}(t)(i=1,2, \ldots)$ in $\mathscr{D}(L)$ and seek approximate solutions $f_{i}(t)$ $(i=1,2, \ldots)$ of the form

$$
f_{i}(t)=\sum_{j=1}^{i} a_{j}^{i} \xi_{j}(t)
$$

where the constants $a_{j}^{i}$ are determined from the system of linear equations

$$
\sum_{j=1}^{i}\left(L \xi_{j}, L \xi_{l}\right) a_{j}^{i}=\left(g_{0}, L \xi_{l}\right), \quad l=1, \ldots, i .
$$

This choice of the constants $a_{j}^{i}$ corresponds to minimizing the quantity $\left\|L f_{i}-g_{0}\right\|^{2}$.

The basic problem encountered in using this method is the construction of the functions $\xi_{i}(t)(i=1,2, \ldots)$. They must guarantee the solvability of equation (3) and the convergence of the approximate solutions (2) to a solution of equation (1). Mihlin [6, p. 493] solves this problem by assuming that (A) the $\xi_{i}(t)$ satisfy a completeness condition, (B) equation (1) is solvable, and (C) the operator $L$ has a bounded inverse.

In this paper we present a method for constructing the approximating sequence $\xi_{i}(t)(i=1,2, \ldots)$ without the restriction that $L$ be invertible; this requires a discussion of existence and uniqueness for the boundary value problem (1). The key to the method is to consider the space $H^{n}[a, b]$ under an appropriate topology.

In $\S 2$ we introduce the strong topology for the space $H^{n}[a, b]$ and examine the notion of completeness with respect to this topology. The notion of a boundary value is explored in $\S 3$, where a characterization is established which is particularly useful from a computational point of view. 
The approximation scheme is developed in $\$ 4$, and error estimates are obtained. For the differential operator $L$ we construct a right inverse operator which is similar to the right inverse operator $H$ constructed in [4]. In $\$ 5$ we modify the method to study nonhomogeneous boundary conditions. We shall examine numerical applications of the method in another paper.

2. The space $H^{n}[a, b]$. For the space $H^{n}[a, b]$ we define a norm by

$$
|f|_{*}=\sum_{i=0}^{n-1} \max _{a \leqq t \leqq b}\left|f^{(i)}(t)\right|+\left\|f^{(n)}\right\| \quad \text { for } f \in H^{n}[a, b] .
$$

Under this norm $H^{n}[a, b]$ becomes a Banach space [3, p. 1296]. We assert that we can induce this topology by means of other equivalent norms, in fact, by means of inner products which induce equivalent norms.

Let $T_{1}(\tau)$ be the linear operator in $S$ defined by

$$
\mathscr{D}\left(T_{1}(\tau)\right)=H^{n}[a, b], \quad T_{1}(\tau) f=\tau f .
$$

It is well known that $T_{1}(\tau)$ is a closed densely-defined linear operator in $S$ with $\mathscr{R}\left(T_{1}(\tau)\right)=S$ and $\operatorname{dim} \mathscr{N}\left(T_{1}(\tau)\right)=n$. Also, by restricting $T_{1}(\tau)$ to the subspace of $\mathscr{D}\left(T_{1}(\tau)\right)$ consisting of those functions which are orthogonal to $\mathscr{N}\left(T_{1}(\tau)\right)$, we obtain a 1-1 closed operator whose inverse

$$
H_{1}(\tau)=\left[T_{1}(\tau) \mid \mathscr{D}\left(T_{1}(\tau) \cap \mathscr{N}\left(T_{1}(\tau)\right)^{\perp}\right]^{-1}\right.
$$

is a 1-1 completely continuous linear operator defined on all of $S$. In [4] it is shown that $H_{1}(\tau)$ is an integral operator, and if we let $P: S \rightarrow S$ be the orthogonal projection onto the subspace $\mathscr{N}\left(T_{1}(\tau)\right)$, then

$$
T_{1}(\tau) H_{1}(\tau) f=f \text { for all } f \in S
$$

and

$$
H_{1}(\tau) T_{1}(\tau) f=f-P f \text { for all } f \in \mathscr{D}\left(T_{1}(\tau)\right) \text {. }
$$

Consider the inner product on $H^{n}[a, b]$ defined by

$$
(f, g)_{\tau}=(f, g)+(\tau f, \tau g) \text { for } f, g \in H^{n}[a, b],
$$

and let $|f|_{\tau}=(f, f)_{\tau}^{1 / 2}$ denote the associated norm. Because $T_{1}(\tau)$ is a closed operator, it follows that $H^{n}[a, b]$ is a Hilbert space under the inner product $(f, g)_{\tau}$.

THEOREM 1. The norms $|f|_{*}$ and $|f|_{\tau}$ are equivalent norms for $H^{n}[a, b]$.

Proof. See [10, p. 431] or [3, pp. 1296-1297].

The theorem shows that there are many equivalent norms which can be used to induce the topology of the Banach space $\left\langle H^{n}[a, b],|f|_{*}\right\rangle$, in fact, there is an equivalent norm corresponding to each $n$th order formal differential operator. We refer to this topology as the strong topology for $H^{n}[a, b]$ to contrast it to the induced $L^{2}$-topology, denoting convergence in the strong topology by $f_{i} \stackrel{s}{\rightarrow} f$. 
Strong convergence is merely uniform convergence of the first $(n-1)$ derivatives on $[a, b]$ together with $L^{2}$-convergence for the $n$th derivatives. In the sequel it will be particularly useful to consider $H^{n}[a, b]$ under the strong topology induced by the inner product determined by the formal differential operator $\tau_{0}=d^{n} / d t^{n}$; we let $[f, g]$ and $|f|$ denote the corresponding inner product and norm, i.e., $[f, g]$ $=(f, g)+\left(f^{(n)}, g^{(n)}\right)$ for $f, g \in H^{n}[a, b]$ and $|f|=[f, f]^{1 / 2}$ for $f \in H^{n}[a, b]$.

Definition. A sequence of functions $\xi_{i}(t)(i=1,2, \ldots)$ in $H^{n}[a, b]$ is complete if the subspace $\left\langle\xi_{1}, \xi_{2}, \ldots\right\rangle$ spanned by the functions is dense in $H^{n}[a, b]$ under the strong topology.

Note that this definition of completeness depends only on the topology of $H^{n}[a, b]$ and is independent of any particular inner product which might be used to induce the topology. In the remainder of this section we establish simple methods for constructing complete sequences in $H^{n}[a, b]$.

Let $T_{0}=T_{1}\left(\tau_{0}\right)$ and $H_{0}=H_{1}\left(\tau_{0}\right)$, and let $P_{0}: S \rightarrow S$ be the orthogonal projection onto the subspace $\mathscr{N}\left(T_{0}\right)=\left\langle 1, t, \ldots, t^{n-1}\right\rangle$. From equations (4) and (5) we have

and

$$
\frac{d^{n}}{d t^{n}}\left[H_{0} f\right]=f \text { for all } f \in S
$$

We use these two equations to prove the following theorem.

THEOREM 2. If $\eta_{i}(t)(i=1,2, \ldots)$ is a sequence of linearly independent functions in $S$ whose linear span $\left\langle\eta_{1}, \eta_{2}, \ldots\right\rangle$ is dense in $S$, then the functions $1, t, \ldots, t^{n-1}$, $H_{0} \eta_{1}(t), H_{0} \eta_{2}(t), \ldots$ form a complete linearly independent sequence in $H^{n}[a, b]$.

Proof. The linear independence follows from the facts that $H_{0}$ is $1-1$ and $\mathscr{R}\left(H_{0}\right)$ is orthogonal to $\mathscr{N}\left(T_{0}\right)$. For the completeness take any $f \in H^{n}[a, b]$. We can choose a sequence of functions $g_{i}(i=1,2, \ldots)$ in $\left\langle\eta_{1}, \eta_{2}, \ldots\right\rangle$ such that $g_{i} \rightarrow f^{(n)}$. Let $f_{i}=P_{0} f+H_{0} g_{i}$ for $i=1,2, \ldots$ Clearly $f_{i} \in\left\langle 1, t, \ldots, t^{n-1}, H_{0} \eta_{1}, H_{0} \eta_{2}, \ldots\right\rangle$ for $i=1,2, \ldots$, and by equation (7) and the continuity of $H_{0}$ we have

$$
f=P_{0} f+H_{0}\left[f^{(n)}\right]=P_{0} f+\lim _{i} H_{0} g_{i}
$$

or $f_{i} \rightarrow f$ in $S$. Also, from equation (6) we have $f_{i}^{(n)}=g_{i}$ for $i=1,2, \ldots$, and hence, $f_{i}^{(n)} \rightarrow f^{(n)}$ in $S$. Thus $\left|f_{i}-f\right| \rightarrow 0$ as $i \rightarrow \infty$, or $f_{i} \stackrel{s}{\rightarrow} f$ in $H^{n}[a, b]$. This completes the proof.

EXAmPle 1. We assert the sequence $1, t, t^{2}, \ldots$ is complete in $H^{n}[a, b]$. From equation (7) we have

$$
\begin{aligned}
H_{0}\left(t^{i}\right)= & {[(i+n)(i+n-1) \cdots(i+1)]^{-1} t^{i+n} } \\
& -P_{0}\left([(i+n)(i+n-1) \cdots(i+1)]^{-1} t^{i+n}\right)
\end{aligned}
$$

for $i=0,1, \ldots$, and these functions are polynomials. Thus, the functions 1 , $t, \ldots, t^{n-1}, H_{0}(1), H_{0}(t), \ldots$ belong to the subspace $\left\langle 1, t, t^{2}, \ldots\right\rangle$, and the assertion follows from Theorem 2. Moreover, suppose we apply the Gram-Schmidt 
process to the sequence $1, t, t^{2}, \ldots$ to obtain an orthonormal sequence of polynomials $\phi_{1}(t), \phi_{2}(t), \ldots$ in $S$. In the proof of the last theorem for any $f \in H^{n}[a, b]$ we have $f^{(n)}=\sum_{i=1}^{\infty}\left(f^{(n)}, \phi_{i}\right) \phi_{i}$, and hence, for the functions $g_{i}$ and $f_{i}$ we can use $g_{i}=\sum_{j=1}^{i}\left(f^{(n)}, \phi_{j}\right) \phi_{j}$ and

$$
f_{i}=\sum_{j=1}^{n}\left(f, \phi_{j}\right) \phi_{j}+\sum_{j=1}^{i}\left(f^{(n)}, \phi_{j}\right) H_{0} \phi_{j}
$$

for $i=1,2, \ldots$ We observe that the functions $f_{i}(t)$ are polynomials, which are easily calculated using (8), and that $f_{i} \stackrel{s}{\rightarrow} f$ in $H^{n}[a, b]$. This yields a simple method of approximating functions in $H^{n}[a, b]$ in terms of the strong topology by means of polynomials.

EXAMPLE 2. Let $S=L^{2}[0,2 \pi]$ and $n=2$. By Theorem 2 the sequence of functions $1, t, H_{0}(1), H_{0}(\sin t), H_{0}(\cos t), \ldots, H_{0}(\sin m t), H_{0}(\cos m t), \ldots$ is a complete linearly independent sequence in $H^{2}[0,2 \pi]$. These functions are easily calculated:

$$
\begin{aligned}
H_{0}(1) & =(1 / 2) t^{2}-\pi t+(1 / 3) \pi^{2}, \\
H_{0}(\sin m t) & =-\left(1 / m^{2}\right) \sin m t-\left(3 / m^{3} \pi^{2}\right)(t-\pi),
\end{aligned}
$$

and

$$
H_{0}(\cos m t)=-\left(1 / m^{2}\right) \cos m t
$$

for $m=1,2, \ldots$. Since these functions belong to the subspace spanned by the sequence $1, t, t^{2}, \sin t, \cos t, \ldots$, we conclude that the sequence of functions $1, t, t^{2}, \sin t, \cos t, \ldots, \sin m t, \cos m t, \ldots$ is complete in $H^{2}[0,2 \pi]$. This argument can be modified for arbitrary intervals $[a, b]$ and arbitrary $n$.

The significance of Theorem 2 is that it yields a useful method for constructing complete sequences in $H^{n}[a, b]$. This is a consequence of the simple structure of the operator $H_{0}$ described in equation (7). To calculate $H_{0} f$ for any $f \in S$, we merely integrate $f(t)$ a total of $n$ times and then subtract off the projection on the subspace $\left\langle 1, t, \ldots, t^{n-1}\right\rangle$. Note that this construction is entirely independent of the formal differential operator $\tau$.

3. Boundary values. We begin this section by summarizing some well-known facts concerning boundary values [3, pp. 1297-1304]. Let $H_{0}^{n}[a, b]$ denote the subspace of $H^{n}[a, b]$ consisting of all functions which vanish outside some compact subset of the open interval $(a, b)$. A boundary value on $H^{n}[a, b]$ is a linear functional $B$ from $H^{n}[a, b]$ into the real numbers which is continuous under the strong topology with $B(f)=0$ for all $f \in H_{0}^{n}[a, b]$. Let $\mathscr{B}_{n}$ denote the space of all boundary values. The following results relate this notion of a boundary value to the classical one:

(a) The space $\mathscr{B}_{n}$ of boundary values is a $2 n$-dimensional real linear space.

(b) The linear functionals $A^{i}(f)=f^{(i)}(a), i=0,1, \ldots, n-1$, and $B^{i}(f)=f^{(i)}(b)$, $i=0,1, \ldots, n-1$, are boundary values which form a basis for $\mathscr{B}_{n}$. 
Given a boundary value $B$, by the Riesz Representation Theorem there exists a unique function $g \in H^{n}[a, b]$ such that

$$
B(f)=[f, g] \text { for all } f \in H^{n}[a, b] .
$$

We want to determine $g$ explicitly. Proceeding formally we must have

$$
\begin{aligned}
B(f) & =\int_{a}^{b} f g+\int_{a}^{b} f^{(n)} g^{(n)} \\
& \left.=\int_{a}^{b} f g+f^{(n-1)} g^{(n)}\right]_{a}^{b}-\int_{a}^{b} f^{(n-1)} g^{(n+1)} \\
& \left.\left.=\int_{a}^{b} f g+f^{(n-1)} g^{(n)}\right]_{a}^{b}-f^{(n-2)} g^{(n+1)}\right]_{a}^{b}+\int_{a}^{b} f^{(n-2)} g^{(n+2)}
\end{aligned}
$$

or

$$
\left.B(f)=\int_{a}^{b} f\left[g+(-1)^{n} g^{(2 n)}\right]+\sum_{j=1}^{n}(-1)^{j+1} f^{(n-j)}(t) g^{(n+j-1)}(t)\right]_{a}^{b}
$$

for all $f \in H^{n}[a, b]$. This implies that the integral in equation (11) is equal to zero for all $f \in H_{0}^{n}[a, b]$, and hence, the function $g$ is a solution of the differential equation

$$
g^{(2 n)}+(-1)^{n} g=0 .
$$

Let $\mathscr{A}_{n}$ denote the $2 n$-dimensional solution space for the differential equation (12). Then for any function $g \in \mathscr{A}_{n}$ we can use the above argument to show that

$$
[f, g]=\sum_{i=0}^{n-1} f^{(i)}(a)\left[(-1)^{n-i} g^{(2 n-i-1)}(a)\right]+\sum_{i=0}^{n-1} f^{(i)}(b)\left[(-1)^{n-i-1} g^{(2 n-i-1)}(b)\right]
$$

for all $f \in H^{n}[a, b]$, and hence, each function $g \in \mathscr{A}_{n}$ determines a unique boundary value $B_{g}$ given by $B_{g}(f)=[f, g]$ for $f \in H^{n}[a, b]$. Equation (13) gives an explicit representation of $B_{g}$ in the classical form.

Consider the mapping $T: \mathscr{A}_{n} \rightarrow \mathscr{B}_{n}$ defined by $T g=B_{g}$ for all $g \in \mathscr{A}_{n}$. Clearly $T$ is a linear transformation, and if $T g=0$, then $[f, g]=0$ for all $f \in H^{n}[a, b]$, and in particular, $[g, g]=0$ or $g=0$. Therefore, $T$ is a vector space isomorphism between the $2 n$-dimensional linear spaces $\mathscr{A}_{n}$ and $\mathscr{B}_{n}$. We summarize these results as a theorem.

THEOREM. 3. If $B$ is a boundary value on $H^{n}[a, b]$ given in classical form by

$$
B(f)=\sum_{i=0}^{n-1} \alpha_{i} f^{(i)}(a)+\sum_{i=0}^{n-1} \beta_{i} f^{(i)}(b)
$$

for $f \in H^{n}[a, b]$, then there exists a unique function $g \in H^{n}[a, b]$ such that $B(f)$ $=[f, g]$ for all $f \in H^{n}[a, b]$. Moreover, the function $g(t)$ is the unique solution of the 
boundary value problem

$$
\begin{aligned}
g^{(2 n)}+(-1)^{n} g & =0, & & \\
g^{(2 n-i-1)}(a) & =(-1)^{n-i} \alpha_{i}, & & i=0,1, \ldots, n-1, \\
g^{(2 n-1-1)}(b) & =(-1)^{n-i-1} \beta_{i}, & & i=0,1, \ldots, n-1 .
\end{aligned}
$$

Conversely, if $g(t)$ is a solution of the differential equation $g^{(2 n)}+(-1)^{n} g=0$, then $g$ determines a boundary value $B$ on $H^{n}[a, b]$ by the defining equation $B(f)=[f, g]$ for all $f \in H^{n}[a, b]$, which is expressed in classical form by equation (13).

4. The approximation scheme. We are ready to consider the boundary value problem

$$
L f=g_{0},
$$

where $L$ is the differential operator determined by the $n$th order formal differential operator $\tau$ and the linearly independent boundary values $B_{1}, \ldots, B_{k}$. The problems of existence and uniqueness are treated by the following well-known results:

(a) There exists a solution to equation (1) iff the function $g_{0}$ is orthogonal to $\mathscr{N}\left(L^{*}\right)$.

(b) A solution to equation (1) is unique iff $\mathscr{N}(L)=\{0\}$.

In most applications it is assumed that $k=n$ and that the homogeneous problem $L f=0$ has only the trivial solution; in this case we have both existence and uniqueness for equation (1). Henceforth, we shall assume that $g_{0}$ is orthogonal to $\mathscr{N}\left(L^{*}\right)$, so we have at least the existence of solutions to equation (1).

Using Theorem 3 we choose functions $g_{1}, \ldots, g_{k}$ in $H^{n}[a, b]$ such that

$$
B_{i}(f)=\left[f, g_{i}\right] \text { for all } f \in H^{n}[a, b], \quad i=1, \ldots, k .
$$

Note that $\mathscr{D}(L)$ is the orthogonal complement of the subspace $\left\langle g_{1}, \ldots, g_{k}\right\rangle$ in the Hilbert space $H^{n}[a, b]$ under the inner product $[f, g]$. Assume that $\operatorname{dim} \mathscr{N}(L)=p$, and choose a basis $\theta_{1}, \ldots, \theta_{p}$ for $\mathscr{N}(L)$. Let

$$
D(L)=\left\{f \in \mathscr{D}(L) \mid\left[f, \theta_{i}\right]=0, i=1, \ldots, p\right\} .
$$

We note that $D(L)$ is the orthogonal complement of the subspace $\left\langle g_{1}, \ldots, g_{k}\right.$, $\theta_{1}, \ldots, \theta_{p}>$ in $H^{n}[a, b]$ under the inner product $[f, g]$.

Consider the restriction $L_{0}=L \mid D(L)$. If $L_{0} f=0$, then $f \in \mathscr{N}(L)$ with $\left[f, \theta_{i}\right]=0$ for $i=1, \ldots, p$ or $f=0$, and hence, $L_{0}$ is a $1-1$ operator. Clearly $\mathscr{R}\left(L_{0}\right)=\mathscr{R}(L)$, which is a closed subspace of $S$. Therefore, the operator $H=L_{0}^{-1}$ exists as a linear operator from $\mathscr{R}(L)$ into $H^{n}[a, b]$. Since $L_{0}$ is continuous from the induced strong topology on $D(L)$ to the induced $L^{2}$-topology on $\mathscr{R}(L)$, there exists a constant $\gamma>0$ such that

$$
|H f|_{*} \leqq \gamma\|f\| \quad \text { for all } f \in \mathscr{R}(L)
$$

In a manner analogous to that used in [4], we can establish an integral representation for $H$; we will not use this result. 
In the subspace $D(L)$ the boundary value problem (1) has a unique solution $f_{0}=H g_{0}$. We are going to construct a sequence of approximate solutions $f_{i}(t)$ $(i=1,2, \ldots)$ which converge to $f_{0}$ and obtain error estimates.

In terms of the inner product $[f, g]$ let $Q$ be the orthogonal projection of $H^{n}[a, b]$ onto the subspace $\left\langle g_{1}, \ldots, g_{k}, \theta_{1}, \ldots, \theta_{p}\right\rangle$. The operator $I-Q$ then becomes the orthogonal projection of $H^{n}[a, b]$ onto $D(L)$. We observe that

$$
\begin{array}{ll}
L H f=f & \text { for all } f \in \mathscr{R}(L), \\
H L f=(I-Q) f & \text { for all } f \in \mathscr{D}(L) .
\end{array}
$$

Let $\rho_{i}(t)(i=1,2, \ldots)$ be a complete linearly independent sequence of functions in $H^{n}[a, b]$, and let $\xi_{i}(t)=\rho_{i}(t)-Q \rho_{i}(t)$ for $i=1,2, \ldots$ With no loss of generality we can assume that the sequence $\xi_{i}(t)(i=1,2, \ldots)$ is linearly independent, for otherwise, we can pass to an appropriate subsequence obtained by deleting at most $k+p$ of the $\xi_{i}(t)$. Note that $\xi_{i}(t) \in D(L) \subset \mathscr{D}(L)$ for $i=1,2, \ldots$ Let $\eta_{i}(t)=\tau \xi_{i}(t)$ for $i=1,2, \ldots$ This new sequence is also linearly independent because $\tau$ is $1-1$ on $D(L)$, and clearly

$$
L \xi_{i}=\eta_{i} \quad \text { and } H \eta_{i}=\xi_{i} \quad \text { for } i=1,2, \ldots
$$

Because the operator $I-Q$ is continuous on $H^{n}[a, b]$ under the strong topology, it follows immediately that the subspace $\left\langle\xi_{1}, \xi_{2}, \ldots\right\rangle$ is dense in $D(L)$ under the strong topology. We assert that the subspace $\left\langle\eta_{1}, \eta_{2}, \ldots\right\rangle$ is dense in $\mathscr{R}(L)$ under the $L^{2}$-topology. Take any function $g \in \mathscr{R}(L)$. We know that there exists a unique function $f \in D(L)$ such that $L f=g$, and by the above remark we can choose a sequence of functions $f_{i}(i=1,2, \ldots)$ in $\left\langle\xi_{1}, \xi_{2}, \ldots\right\rangle$ such that $f_{i} \stackrel{s}{\rightarrow} f$. Let $g_{i}$ $=L f_{i}=\tau f_{i}$ for $i=1,2, \ldots$ Clearly $g_{i} \in\left\langle\eta_{1}, \eta_{2}, \ldots\right\rangle$ for $i=1,2, \ldots$, and from the strong convergence of the $f_{i}$ to $f$ we conclude that $\tau f_{i} \rightarrow \tau f$ or $g_{i} \rightarrow g$.

Working in the $L^{2}$-inner product, we apply the Gram-Schmidt process to the sequence $\eta_{i}(i=1,2, \ldots)$ to form a complete orthonormal sequence $\omega_{i}(i=1,2, \ldots)$ for the closed subspace $\mathscr{R}(L)$. Let $\phi_{i}=H \omega_{i} \in D(L)$ for $i=1,2, \ldots$ If $\omega_{i}$ $=\sum_{j=1}^{i} a_{i j} \eta_{j}$ for $i=1,2, \ldots$, then from equation (18) we have $\phi_{i}=\sum_{j=1}^{i} a_{i j} \xi_{j}$ for $i=1,2, \ldots$, and hence, as we inductively construct the functions $\omega_{i}$, we can simultaneously construct the functions $\phi_{i}$ in a manner which avoids any use of the operator $H$. Also,

$$
L \phi_{i}=\omega_{i} \text { and } H \omega_{i}=\phi_{i} \text { for } i=1,2, \ldots
$$

For $i=1,2, \ldots$, let $P_{i}: S \rightarrow S$ and $Q_{i}: \mathscr{D}(L) \rightarrow \mathscr{D}(L)$ be the linear operators defined by

and

$$
P_{i} f=\sum_{j=1}^{i}\left(f, \omega_{j}\right) \omega_{j} \quad \text { for } f \in S
$$

$$
Q_{i} f=\sum_{j=1}^{i}\left(L f, \omega_{j}\right) \phi_{j} \quad \text { for } f \in \mathscr{D}(L) .
$$


Note that $P_{i}$ is the orthogonal projection from $S$ onto the subspace $\left\langle\omega_{1}, \ldots, \omega_{i}\right\rangle$, and

$$
f=\sum_{i=1}^{\infty}\left(f, \omega_{i}\right) \omega_{i}=\lim _{i} P_{i} f
$$

for all $f \in \mathscr{R}(L)$. The operator $Q_{i}$ is a projection from $\mathscr{D}(L)$ onto the subspace $\left\langle\phi_{1}, \ldots, \phi_{i}\right\rangle$, and in fact, $Q_{i}=H P_{i} L$. Also, we have

$$
P_{i} L f=L Q_{i} f \text { for all } f \in \mathscr{D}(L) .
$$

It can be shown that $Q_{i}$ is continuous on $\mathscr{D}(L)$ under the strong topology but not under the $L^{2}$-topology.

We now use the projection operators $Q_{i}$ to construct approximate solutions to the solution $f_{0}=H g_{0}$ of the boundary value problem (1). Let $f_{i}=Q_{i} f_{0}$ for $i=1,2$, (.., i.e.,

$$
f_{i}=\sum_{j=1}^{i}\left(g_{0}, \omega_{j}\right) \phi_{j} \quad \text { for } i=1,2, \ldots
$$

Now $L f_{i}=\sum_{j=1}^{i}\left(g_{0}, \omega_{j}\right) \omega_{j}$ for $i=1,2, \ldots$, and hence, from equation (20) we have $L f_{i} \rightarrow g_{0}=L f_{0}$. Using the continuity of $H$, we conclude that $f_{i} \stackrel{s}{\rightarrow} f_{0}$. In fact, from equation (15) we obtain

$$
\left|f_{i}-f_{0}\right|_{*}=\left|H L f_{i}-H L f_{0}\right|_{*} \leqq \gamma\left\|L f_{i}-g_{0}\right\|,
$$

which yields the error estimate

$$
\left|f_{i}-f_{0}\right|_{*} \leqq \gamma\left\|\sum_{j=1}^{i}\left(g_{0}, \omega_{j}\right) \omega_{j}-g_{0}\right\| \text { for } i=1,2, \ldots
$$

It is possible to give another interpretation to the approximate solutions $f_{i}(t)$. If we apply the operator $P_{i}$ to the equation $L f_{0}=g_{0}$, then from equation (21) we obtain

$$
L f_{i}=P_{i} g_{0} .
$$

Now $L$ is an isomorphism between the subspaces $\left\langle\phi_{1}, \ldots, \phi_{i}\right\rangle$ and $\left\langle\omega_{1}, \ldots, \omega_{i}\right\rangle$, and hence, the function $f_{i}$ is the unique solution in the subspace $\left\langle\phi_{1}, \ldots, \phi_{i}\right\rangle$ to the linear equation (24). But $\left\langle\phi_{1}, \ldots, \phi_{i}\right\rangle=\left\langle\xi_{1}, \ldots, \xi_{i}\right\rangle$ and $\left\langle\omega_{1}, \ldots, \omega_{i}\right\rangle$ $=\left\langle\eta_{1}, \ldots, \eta_{i}\right\rangle$, and hence, if we write $f_{i}$ in the form

$$
f_{i}=\sum_{j=1}^{i} a_{j}^{i} \xi_{j}
$$

then we can determine the coefficients $a_{j}^{i}$ from equation (24):

$$
\sum_{j=1}^{i} a_{j}^{i} L \xi_{j}=\sum_{j=1}^{i}\left(g_{0}, \omega_{j}\right) \omega_{j},
$$


and taking the inner product with $\omega_{l}(l=1, \ldots, i)$ we obtain

$$
\left(\sum_{j=1}^{i} a_{j}^{i} L \xi_{j}-g_{0}, \omega_{l}\right)=0 \text { for } l=1, \ldots, i .
$$

This last equation is equivalent to

or

$$
\left(\sum_{j=1}^{i} a_{j}^{i} L \xi_{j}-g_{0}, \eta_{l}\right)=0 \text { for } l=1, \ldots, i
$$

$$
\sum_{j=1}^{i}\left(L \xi_{j}, L \xi_{l}\right) a_{j}^{i}=\left(g_{0}, L \xi_{l}\right), \quad l=1, \ldots, i .
$$

In equations (25) and (26) we have obtained the equations for the method of least squares, i.e., equations (2) and (3) described in the introduction. The coefficient matrix in equations (26) is nonsingular because the functions $\eta_{1}=L \xi_{1}, \ldots, \eta_{i}$ $=L \xi_{i}$ are linearly independent. The error estimate (23) can be rewritten as

$$
\left|\sum_{j=1}^{i} a_{j}^{i} \xi_{j}-f_{0}\right|_{*} \leqq \gamma\left\|\sum_{j=1}^{i} a_{j}^{i} L \xi_{j}-g_{0}\right\|
$$

for $i=1,2, \ldots$ We summarize these results as a theorem.

THEOREM 4. Assume that $g_{0}$ is orthogonal to $\mathscr{N}\left(L^{*}\right)$ and that the sequence of functions $\xi_{i}(t)(i=1,2, \ldots)$ is constructed as above. Then for each integer $i=1,2, \ldots$ the linear system of equations

$$
\sum_{j=1}^{i}\left(L \xi_{j}, L \xi_{l}\right) a_{j}^{i}=\left(g_{0}, L \xi_{l}\right), \quad l=1, \ldots, i,
$$

has a unique solution $a_{1}^{i}, \ldots, a_{i}^{i}$, and the sequence of functions $f_{i}(t)=\sum_{j=1}^{i} a_{j}^{i} \xi_{j}(t)$, $i=1,2, \ldots$, converges in the strong topology to the solution $f_{0}=H g_{0}$ of the boundary value problem (1). Moreover, the rate of convergence is determined by equation (27).

REMARK. In examining the constructive aspects of the method used to obtain the approximate solutions $f_{i}(t)(i=1,2, \ldots)$, there are two basic problems. First, we assume that $g_{0}$ is orthogonal to $\mathscr{N}\left(L^{*}\right)$. Checking this condition corresponds to solving the homogeneous boundary value problem $L^{*} f=0$, which may be extremely difficult to do. Second, we assume that we can find a basis $\theta_{1}, \ldots, \theta_{p}$ for $\mathscr{N}(L)$; this permits us to compute with the projection operator $Q$, and in particular, to construct the approximating sequence $\xi_{i}(t)(i=1,2, \ldots)$. Obtaining this basis corresponds to solving the homogeneous boundary value problem $L f=0$. As mentioned earlier in most applications we assume that $k=n$ and that $L f=0$ has only the trivial solution, and in this situation these two basic problems do not arise.

5. Nonhomogeneous boundary conditions. Given real numbers $\alpha_{1}, \ldots, \alpha_{k}$, we consider the boundary value problem

$$
\tau f=g_{0}, \quad B_{i}(f)=\alpha_{i}, \quad i=1, \ldots, k .
$$


We can use the methods of the last section to construct approximate solutions for this problem.

Consider the linear system of equations

$$
\sum_{j=0}^{n-1} \alpha_{i j} x_{j}+\sum_{j=0}^{n-1} \beta_{i j} y_{j}=\alpha_{i}, \quad i=1, \ldots, k,
$$

where the coefficients $\alpha_{i j}$ and $\beta_{i j}$ are the coefficients occurring in the classical representations of the boundary values $B_{1}, \ldots, B_{k}$. Since the boundary values are linearly independent, this system has rank $k$, and hence, there exists a solution $\left(x_{0}^{*}, \ldots, x_{n-1}^{*}, y_{0}^{*}, \ldots, y_{n-1}^{*}\right)$. Choose a function $u(t)$ in $H^{n}[a, b]$ such that $u^{(j)}(a)=x_{j}^{*}$ and $u^{(j)}(b)=y_{j}^{*}$ for $j=0,1, \ldots, n-1$; we can utilize interpolation methods to construct $u$. Clearly $B_{i}(u)=\alpha_{i}$ for $i=1, \ldots, k$.

Let $h_{0}=g_{0}-\tau u$, and consider the boundary value problem

or

$$
\tau f=h_{0}, \quad B_{i}(f)=0, \quad i=1, \ldots, k,
$$

$$
L f=h_{0} .
$$

We assume that $h_{0}$ is orthogonal to $\mathscr{N}\left(L^{*}\right)$, and hence, equation (29) has a unique solution $f_{0} \in D(L)$. For equation (29) let $f_{i}(t)(i=1,2, \ldots)$ be the sequence of approximate solutions constructed in $\S 4$. Clearly $f_{i} \in D(L) \subset \mathscr{D}(L)$ for $i=1,2, \ldots$ and $f_{i} \stackrel{s}{\rightarrow} f_{0}$. Let

$$
u_{0}=u+f_{0} \quad \text { and } \quad u_{i}=u+f_{i} \text { for } i=1,2, \ldots
$$

The function $u_{0}(t)$ belongs to $H^{n}[a, b]$ and is a solution of equation (28), while for $i=1,2, \ldots$ we have $u_{i}(t) \in H^{n}[a, b]$ with $B_{j}\left(u_{i}\right)=\alpha_{j}$ for $j=1, \ldots, k$ and $u_{i} \stackrel{s}{\rightarrow} u_{0}$. Also, we obtain the error estimate

$$
\left|u_{i}-u_{0}\right|_{*}=\left|f_{i}-f_{0}\right|_{*} \text { for } i=1,2, \ldots
$$

\section{REFERENCES}

1. P. G. Ciarlet, M. H. Schultz and R. S. Varga, Numerical methods of high-order accuracy for nonlinear boundary value problems. I. One dimensional problem, Numer. Math. 9 (1967), 394-430. MR 36 \#4813.

2. - Numerical methods of high-order accuracy for nonlinear boundary value problems. II. Nonlinear boundary conditions, Numer. Math. 11 (1968), 331-345. MR 37 \#4965.

3. N. Dunford and J. T. Schwartz, Linear operators. I, II, Interscience, New York, 1958, 1963. MR 22 \#8302; MR 32 \#6181.

4. J. Locker, An existence analysis for nonlinear boundary value problems, SIAM J. Appl. Math. 19 (1970), 199-207.

5. - An existence analysis for nonlinear equations in Hilbert space, Trans. Amer. Math. Soc. 128 (1967), 403-413. MR 35 \#5985.

6. S. G. Mihlin, Variational methods in mathematical physics, GITTL, Moscow, 1957; English transl., Macmillan, New York, 1964. MR 22 \#1981; MR 30 \#2712.

7. - Variational methods of solving linear and non-linear boundary value problems, Proc. Conference Differential Equations and their Applications (Prague, 1962), Publ., House Czechoslovak Acad. Sci., Prague; Academic Press, New York, 1963, pp. 77-92. MR 30 \#372. 
8. S. G. Mihlin and K. L. Smolickiī, Approximate methods of solution of differential and integral equations, "Nauka", Moscow, 1965; English transl., Modern Analytic and Computational Methods in Science and Math., vol. 5, American Elsevier, New York, 1967. MR 33 \#855; MR 36 \#1108.

9. W. V. Petryshyn, Direct and iterative methods for the solution of linear operator equations in Hilbert space, Trans. Amer. Math. Soc. 105 (1962), 136-175. MR 26 \#3180.

10. J. Schwartz, Perturbations of spectral operators, and applications. I. Bounded perturbations, Pacific J. Math. 4 (1954), 415-458. MR 16, 144.

11. R. S. Varga, Hermite interpolation-type Ritz methods for two-point boundary value problems, Proc. Sympos. Numerical Solution of Partial Differential Equations (University of Maryland, 1965), Academic Press, New York, 1966, pp. 365-373. MR 34 \#5302.

Colorado State University,

Fort Collins, Colorado 80521 